\title{
NUMERICAL INVESTIGATION OF BORE HOLE FILLING VOLUME IN A COASTAL AREA
}

\author{
Feddy ADONG ${ }^{1}$, Anne-Claire BENNIS ${ }^{1}$, Dominique MOUAZÉ ${ }^{1}$
}

\begin{abstract}
A numerical study is carried out to determine the parameters controlling the filling of a bore hole in Alderney Race. Our final goal is to identify the configuration minimizing the filling. We performed a one-way coupling between a coastal circulation model and a discrete element model for that purpose. Simulations used spherical particles and a monopile technology. We show that: as long as the distance between the hole and the residuals remains smaller than twenty meters, the flow direction plays a negligible role; interaction between moving particles and bottom roughness leads to a slight increase of the filling; the impact of ambient sediments strongly depends on seabed morphology and current effects.
\end{abstract}

Keywords: Tidal energy; Alderney Race; Marine foundations; Sediment transport; Filling volume; Numerical modelling

\section{INTRODUCTION}

In the quest of reducing the carbon fossil energy, tidal energy exploitation is an appealing solution due to its hight possibility of produces energy. However placing turbines in tidal stream is complex and still faces a number of technological barriers like the bore hole filling by drill residuals and ambient sediments. For logistical reasons, the installation phase is carried out a few days after the drilling phase. During this time, the bore hole can be filled by drilling residuals or ambient sediments. To study the parameters controlling this filling, we perform a coupling between a coastal circulation model and a discrete element model, with an application to Alderney Race, and considering spherical particles. The model is described, and used to determine the parameters controlling the filling of one bore hole placed in a specific region of Alderney Race. The results are analysed for different disposition of residuals and initial current direction and intensity. Firstly, only the drilling residuals are considered (no roughness and ambient sediments). We provide numerical simulations to obtain the configuration minimizing the filling. Secondly, this configuration is used to study the effects of bed roughness and ambient sediments on the filling.

\section{NUMERICAL MODELLING}

Hydrodynamical model

This paper focuses on a small region in Alderney Race where tidal converters should be installed. The circulation in this region is calculated using the grid nested approach whose the structure is presented in Figure 1. This procedure allows to enhance the model spatial resolution by the multiplication of nested sub domains (hereafter named ranks). The advantages is to take into account the different dynamics or models in each domain. So, velocity and sea surface elevation are computed using barotropic model on previous coarser ranks and are applied to generate boundary of the 3D finest rank, where the particle sediment transport is simulated. Hydrodynamic fields are computed using the coastal model MARS (Lazure and Dumas, 2008). This model solves the hydrostatic primitive equations using the following-terrain coordinates $(\sigma)$ and the Boussinesq assumption.

\section{Discrete Element Method}

The sediment scale informations (velocity, position and force) are computed using the Discrete Element Method (DEM). It consists in the tracking of each sediment (represented by a sphere) by solving the second Newton's law, expressing the contact forces between particles and potential walls (the seabed). The spheres are set in motion by the hydrodynamical and gravity forces so that the evolution of each particle $\mathrm{p}$ is expressed as:

$$
m_{p} \frac{d \mathbf{u}_{\mathbf{p}}}{d t}=\mathbf{f}_{\mathbf{f}, \mathbf{p}}+\sum_{i=1}^{n_{c}} \mathbf{f}_{\mathbf{c}, \mathbf{p}}+m_{p} \mathbf{g}
$$

where $\mathbf{u}_{\mathbf{p}}$ is the velocity of each particle $\mathrm{p}$ and $n_{c}$ is the number of particles which interact with the particle p. The forces involved are: the inter-particle force between particles $\mathbf{f}_{\mathbf{c}, \mathbf{p}}$ which is defined explicitly using a laws depending on particles overlap; and the external forces composed of gravitational force $m_{p} \mathbf{g}$ (with $\mathbf{g}$ the gravity vector) and the forces applied by the fluid on the sediment $\mathbf{f}_{\mathbf{f}, \mathbf{p}}$. In the present study, for simplification, $\mathbf{f}_{\mathbf{f}, \mathbf{p}}$ is restricted only to dominant forces acting in the filling volume: the drag and the buoyancy forces.

\footnotetext{
${ }^{1}$ M2C Laboratory, CNRS-UNICAEN-UNIROUEN-Normandie Univ, 24 rue des Titteuls
} 

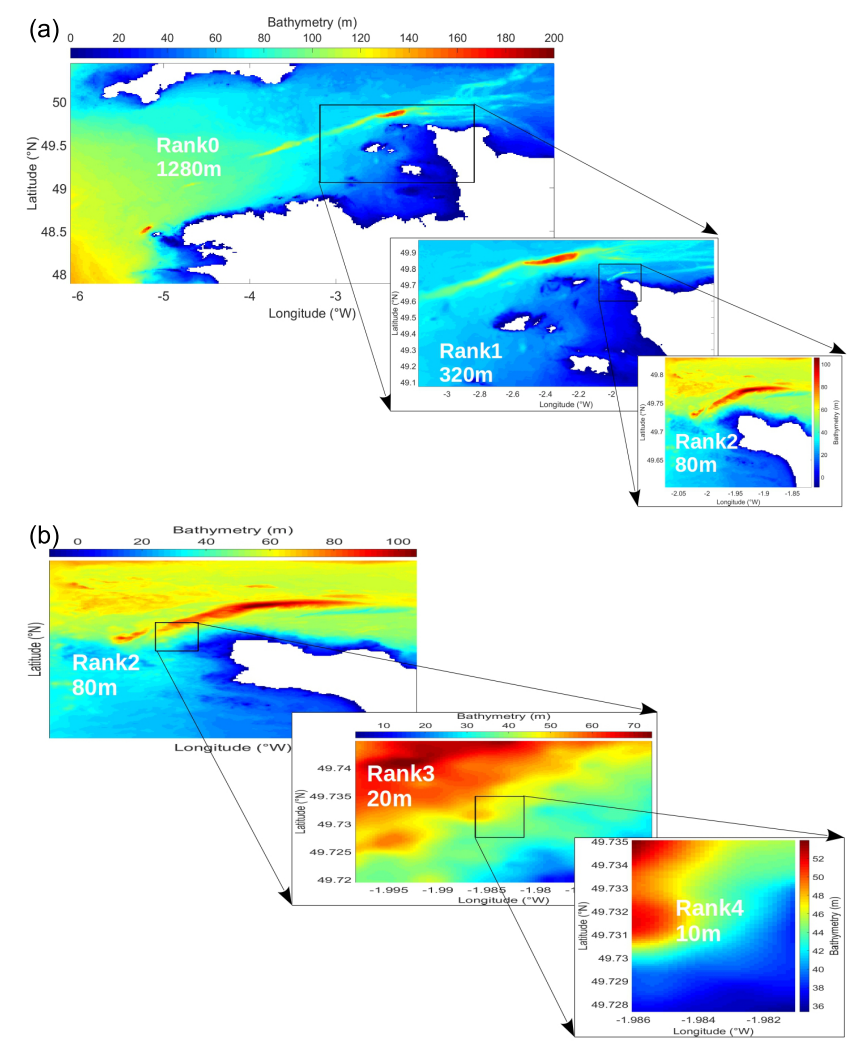

Figure 1: Computational domains used for MARS simulations (ranks 0, 1, 2, 3, 4)

\section{One way coupling}

The forcing is based on the sending of fields at initial time $\left(t=t_{\text {init }}\right)$ and as well as at every forcing time $\left(t=t_{\text {forcing }}\right)$. First, at $t=t_{\text {init }}$, MARS send to the DEM code the water depth $(H)$, the sea surface elevation $(\eta)$ and the fluid velocity $\left(\mathbf{u}_{\mathbf{f}}=(u, v, w)\right) . H$ and $\eta$ are used to compute the index of fluid cell containing the particle whereas the fluid velocity $\left(\mathbf{u}_{\mathbf{f}}\right)$ allows the initialisation of the drag force. At every forcing time ( $t=t_{\text {forcing }}$ ), MARS send $\eta$ and $\mathbf{u}_{\mathbf{f}}$ to the DEM code in order to recalculate the fluid cell index and the drag force, respectively. The forcing time is set to $t=t_{\text {forcing }}=120 \mathrm{~s}$.

Numerical implementation of this procedure has been realized by modifying available functions included in the open source DEM model YADE (Šmilauer et al., 2015). The modifications generalize the work (Maurin et al., 2015) by ensuring the forcing of spherical particle by a three dimensional tidal current over a realistic seabed (Adong et al., in press). The main difficulty lies in the detection of fluid cell containing a sphere due to use of $\sigma$ coordinates. Its require to know the bathymetry value corresponding to the horizontal particle position. The equation of plane of the triangular element containing the particle is employed for that purpose.

\section{APPLICATION TO BORE HOLE FILLING \\ Configuration}

A cylindrical bore hole of diameter $1.2 \mathrm{~m}$ and $10 \mathrm{~m}$ depth is placed at center of rank 4 . The hole parameters are consistent with the public data of the Seagen project. To ensure a reasonable computating time, the flow inside the holes is not modelled and only the buoyancy force is applied in this region. This means that, residuals and sediments will remain trapped inside the hole. At least, 10008 spheres are used to represent the drilling residuals composed of granite. The latter are uniformly arranged in three concentric circles, each containing residuals of different size. The nearest circle of the hole contains 1430 spheres of diameter $d_{p}=4 \mathrm{~cm}$; the second contains 2860 spheres of diameter $d_{p}=2 \mathrm{~cm}$; and the third circle contains 5720 spheres of diameter $d_{p}=1 \mathrm{~cm}$. This disposition allows to obtain three crows of $0.6 \mathrm{~m}$ height. The distance between the nearest circle of the center is $\Delta_{H R}$ as presented in Figure 2. Ambient sediments are 


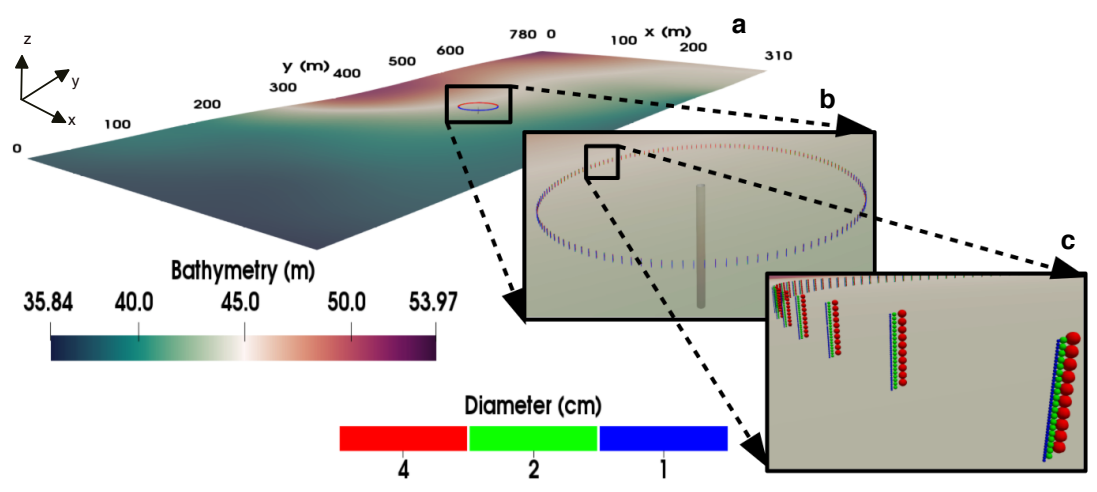

Figure 2: Initial locations of drill residuals around the bore hole (gray cylinder in (b)) and along three concentric circles. The seabed is coloured according to bathymetry (a) and the residuals according to their diameter: $4 \mathrm{~cm}, 2 \mathrm{~cm}$ and $1 \mathrm{~cm}(\mathrm{c})$.

modelled using 10000 additional limestone spherical particles of $5 \mathrm{~cm}$ diameter. At initialization, ambient sediments are randomly generated in a box whose the position and dimensions vary with the tidal current direction (Figure 3).

\section{Filling volume}

The total volume of spheres used to model the drilling residuals is approximately $0,28 \mathrm{~m}^{3}$ (summation over the 10000 spherical particles volume). However this volume underestimates the global volume of drilling residuals which is about of $15,4 \mathrm{~m}^{3}$ (volume of cylinder hole). Therefore, our representation of drilling residual can't be used to exactly quantify the bore hole filling volume. To overcome this difficulty we propose to use this representation to study the parameters controlling the filling. For this purpose, the filling is quantified by calculating the percentage of volume trapped in the hole over the total volume modelled as

$$
V_{\text {trap }}=100 \times \frac{\text { volume trapped in the hole }}{\text { total volume modelled }}
$$

\section{RESULTS AND DISCUSSIONS Tidal dynamic}

Tidal currents are simulated during $T=8$ hours starting from the 19th June 2016 at 00h:00mn:00s, where wave effects are negligible. The period simulated includes tide reversal when the flow direction changes (Figure 4 (a)). Due to the OpenMP parallelization used in YADE, the investigation of filling volume during a full tidal cycle requires an important computational cost and is currently not possible. To overcome this difficulty, the flow effects on the sediment transport are studied by choosing three different dates to initialize the calculation of the fluid forces in YADE. Each date represents specific moment of the tidal cycle as presented in Figure 4. These dates are denoted date $1=T / 5$, when free surface elevation is negative and the flow velocity is Southwestward; date $2=2 T / 5$ when the flow velocity decreases and the current orientation reverses; and date $3=3 T / 5$ when the free surface elevation is positive and the flow velocity is Northeastward.

\section{Influence of $\Delta_{H R}$ parameter}

We start our study with the analysis of the potential effects of the distance $\Delta_{H R}$ on the filling by drilling residuals over a smooth bathymetry. The main result is the reduction of the filling with the increasing of $\Delta_{H R}$. It becomes more prominent for $\Delta_{H R}=20 \mathrm{~m}$, which can be observed in Figure 5(a). Through a comparison of the particle distribution inside the hole, Figure 5(b) shows that this reduction is not only due to one class of particle. Indeed, the residuals are essentially moved far from the hole due to effects of tidal currents and bathymetry variations. So, when the distance $\Delta_{H R}$ is increased, particles have sufficient time to avoid the bore hole and then reduce the filling. We can conclude that the distance $\Delta_{H R}$ and the initial bore hole position are the first keys parameters controlling the filling by drilling residuals. The following results have been obtained using $\Delta_{H R}=20 \mathrm{~m}$ which is the value minimizing the bore hole filling volume. 


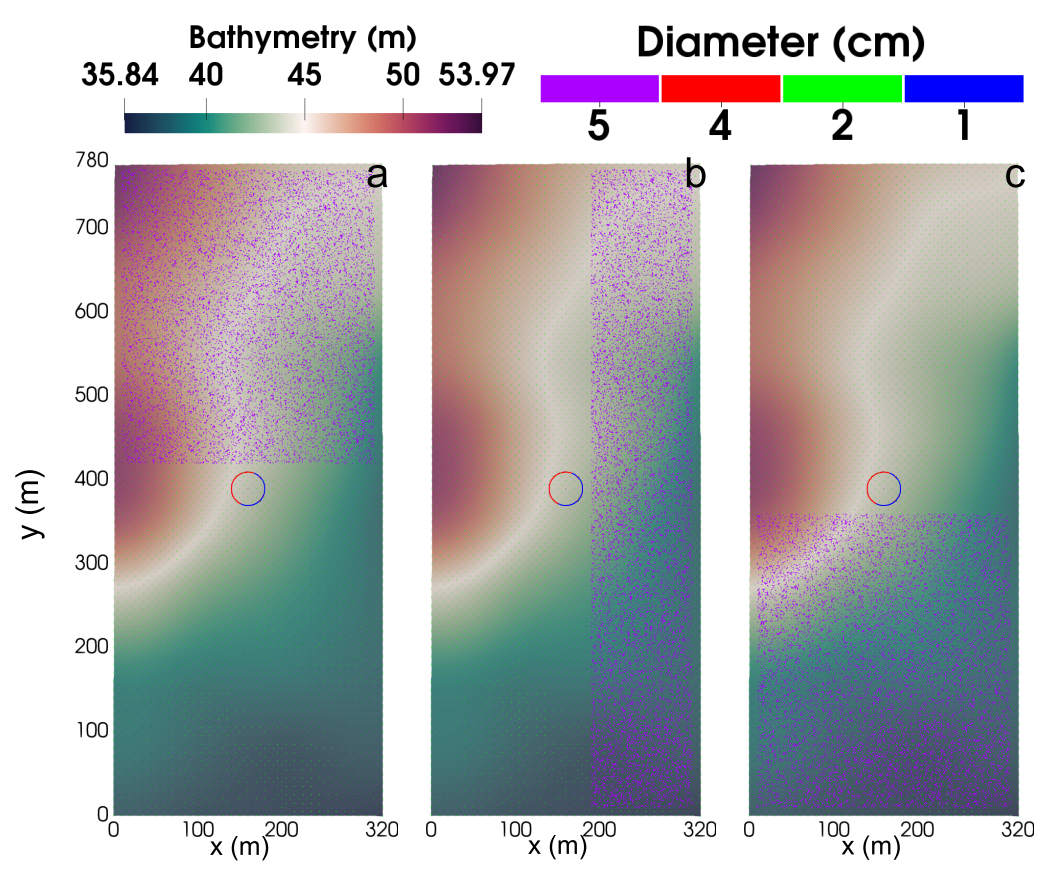

Figure 3: Top view of the initial location of ambient sediments (purple dots) and drill residual (red-greenblue circles at the middle of a, b, c ) at: (a) $t_{\text {init }}=$ date1, (b) $t_{\text {init }}=$ date2, (c) $t_{\text {init }}=$ date3. Particles are drawn according to their diameter from 1 to $5 \mathrm{~cm}$. Coloured contours represent the bathymetry.

\section{Bed roughness}

We now consider a roughened bathymetry whose the bed roughness is generated by granite fixes spherical particles of $2 \mathrm{~cm}$ diameter. The fixed spheres are uniformly arranged on the edges of triangular elements composing the bathymetry and are embedded in the later so that only the half of spheres surface is in contact with the moved sediments. It appears that the contacts between the drilling and the fixed spheres generate a slightly increase of the the filling volume (Figure 6 (a)). In addition the comparison of the particle distribution trapped inside the hole shows that contacts with the fixed sphere affect the filling differently according to the diameter of residuals. Specifically, effects of both seabed morphology and tidal currents direction can be decomposed following three main cases:

- Case1: Combined effects of seabed morphology and Southwestward current (date 1)

- Case2: Dominant effect of seabed morphology (date 2)

- Case3: Opposing effects between the seabed morphology and the Northeastward current (date 3)

For the case1, the particles of $2 \mathrm{~cm}$ and $4 \mathrm{~cm}$ diameter are those which increase the volume trapped. Here the contacts with fixed spheres reorient towards the hole the particles of the diameter greater than the roughnesses size. In particular, the residuals smaller than roughnesses are redirected far from the hole. This may be result by the dissipation of kinetic energy which induces a dominant effect of bathymetry variations. Kinetic energy is dissipated during each contact with the fixes particles and its effect is more visible for smallest particles where kinetic energy is lower. Consequently, the decreasing velocity of the smallest particles leads to increase the role played by the seabed morphology. During the reverse event (case2), the increase of volume trapped is essentially caused by the small particles migration (1cm) towards the hole showing a lower effect of kinetic energy dissipation than for the case1. That leads to create a dominant effect of the seabed morphology on the particle motion. In the case 3, just seabed morphology and the current direction are opposite. In this case, no significant reorientation of particle occurs unlike the case 1 and case2. Kinetic energy dissipation plays a negligible role. 
a
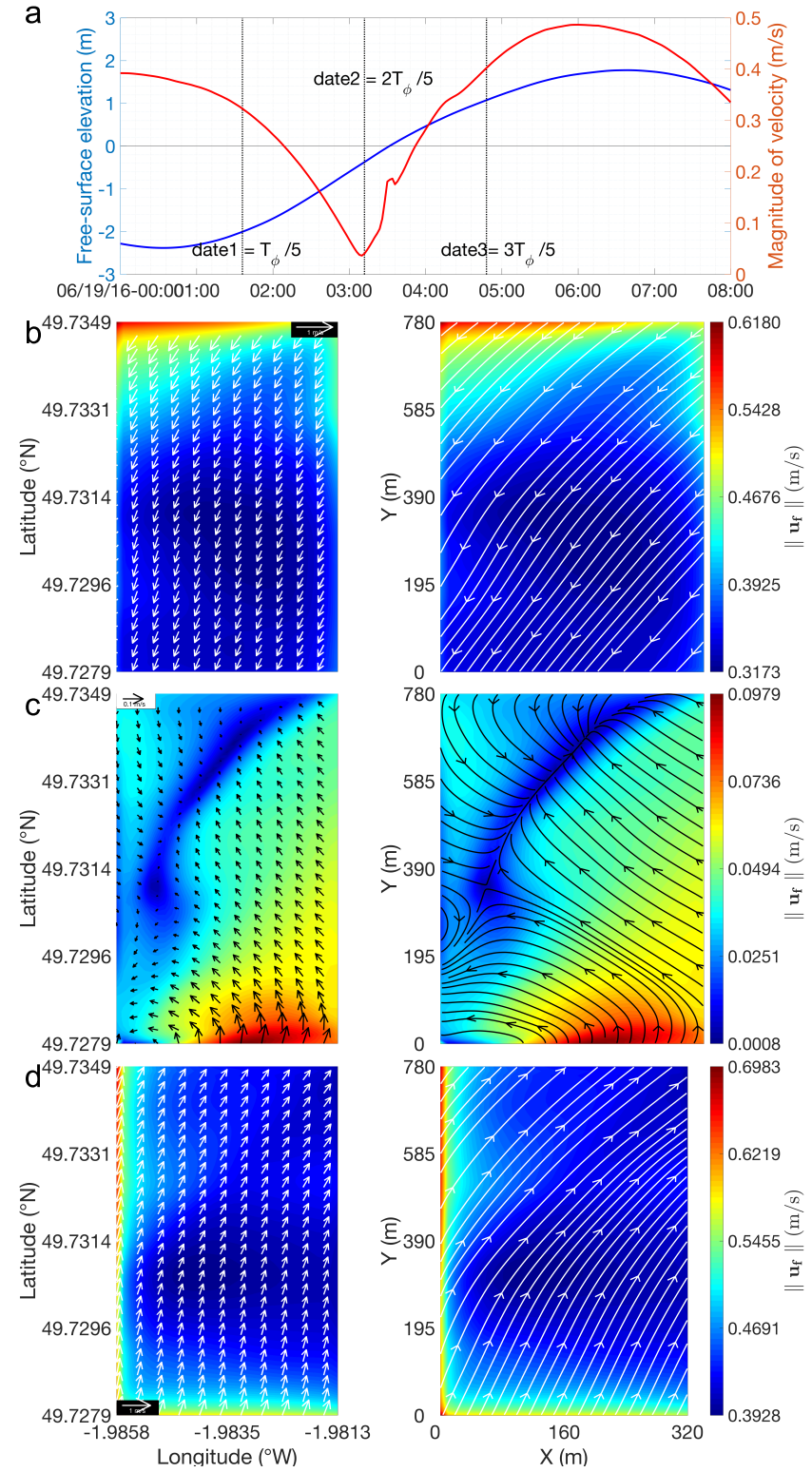

Figure 4: Time evolution of free-surface elevation and magnitude of currents near the seabed (first sigma level) (a). Direction and intensity of tidal currents near the seabed and corresponding streamlines in Cartesian coordinates at date $1(b)$, date 2 (c) and date $3(d)$. 

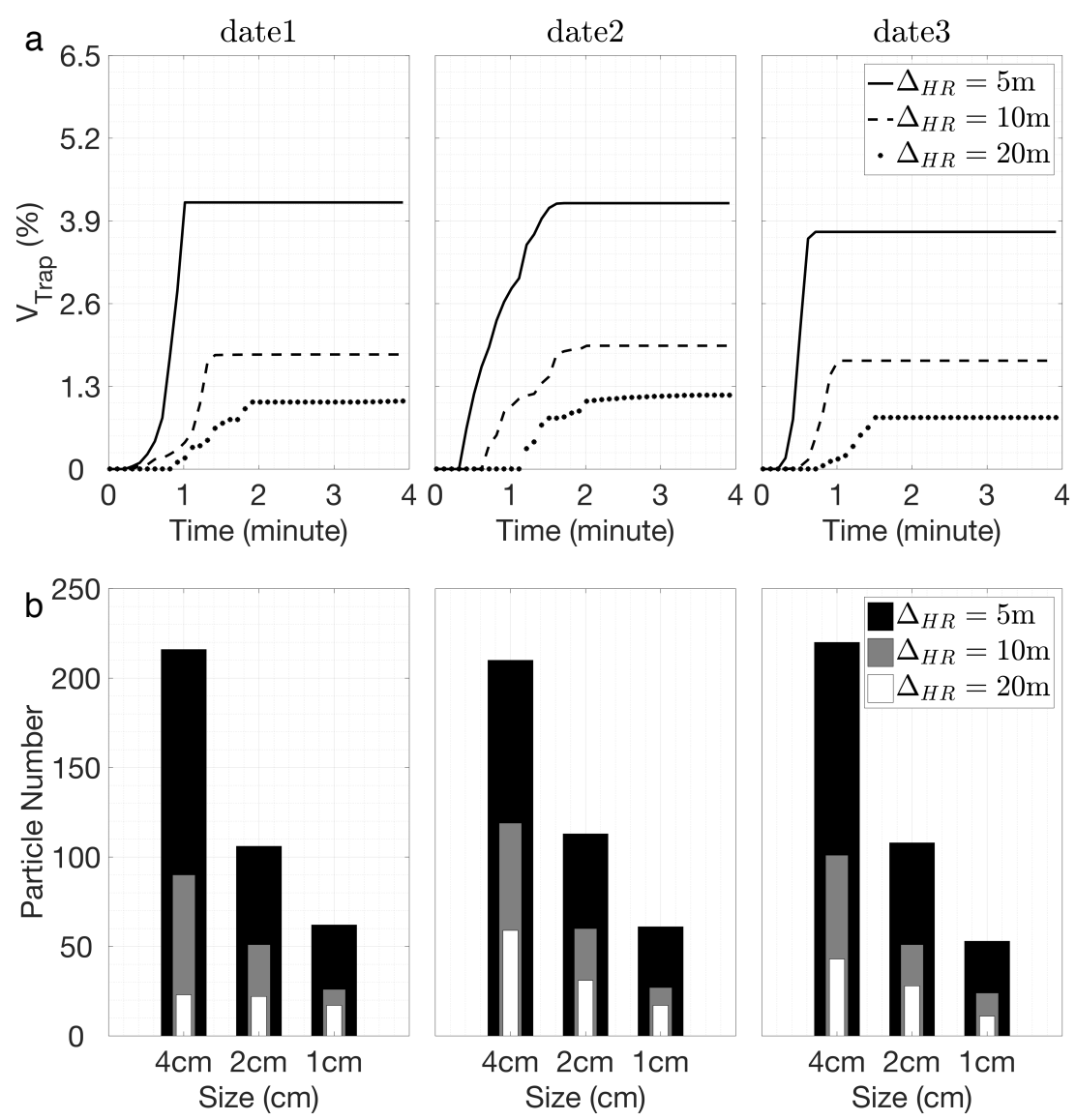

Figure 5: Variation of filling volume with time and final particles distribution inside the hole using $\Delta_{H R}=5 \mathrm{~m}, 10$ and $20 \mathrm{~m}$. 

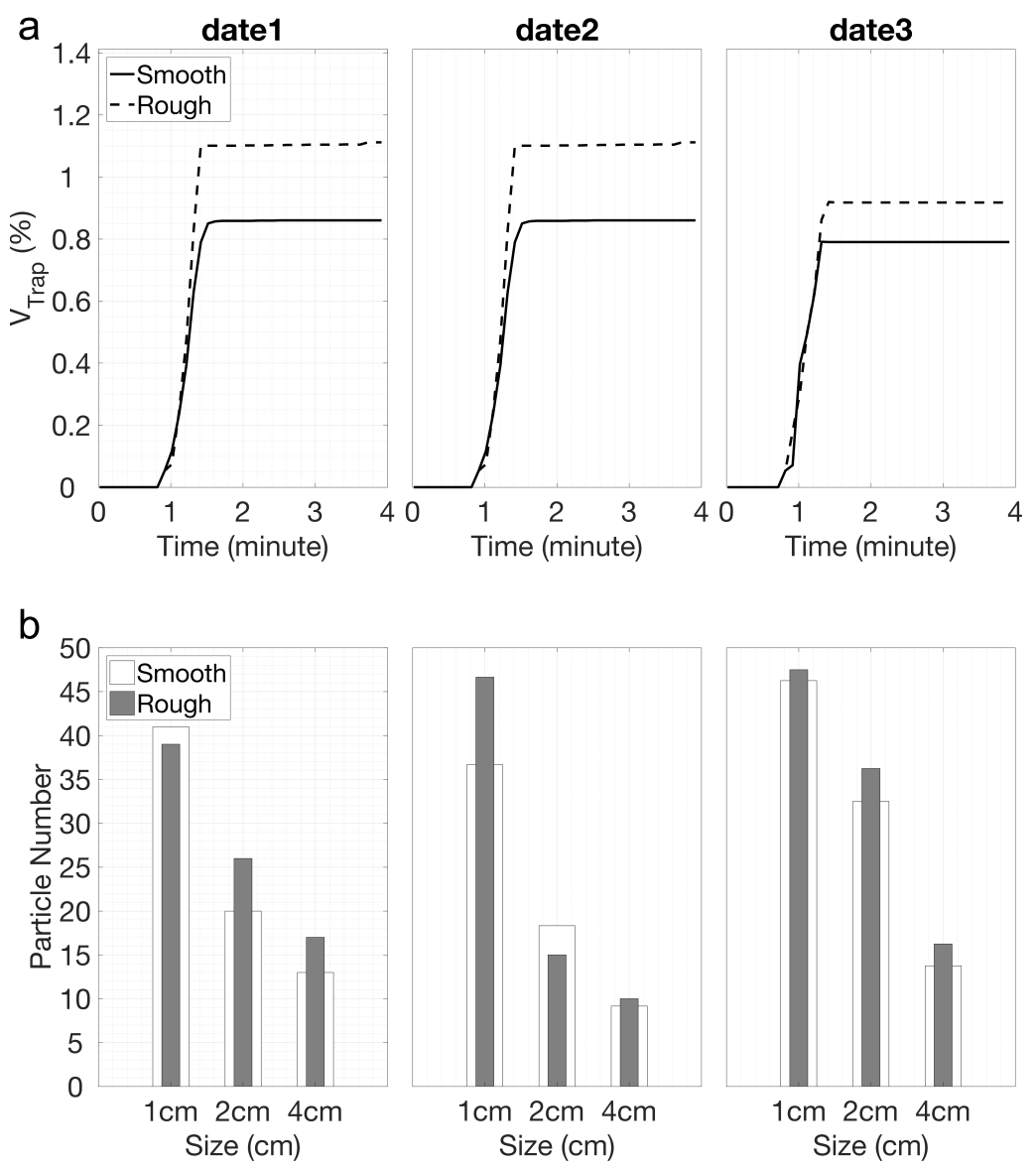

Figure 6: Effect of bed roughness on the filling profiles and the final particles distribution inside the hole. The fixes particles affect the residuals differently according to their size and the tidal current direction. 


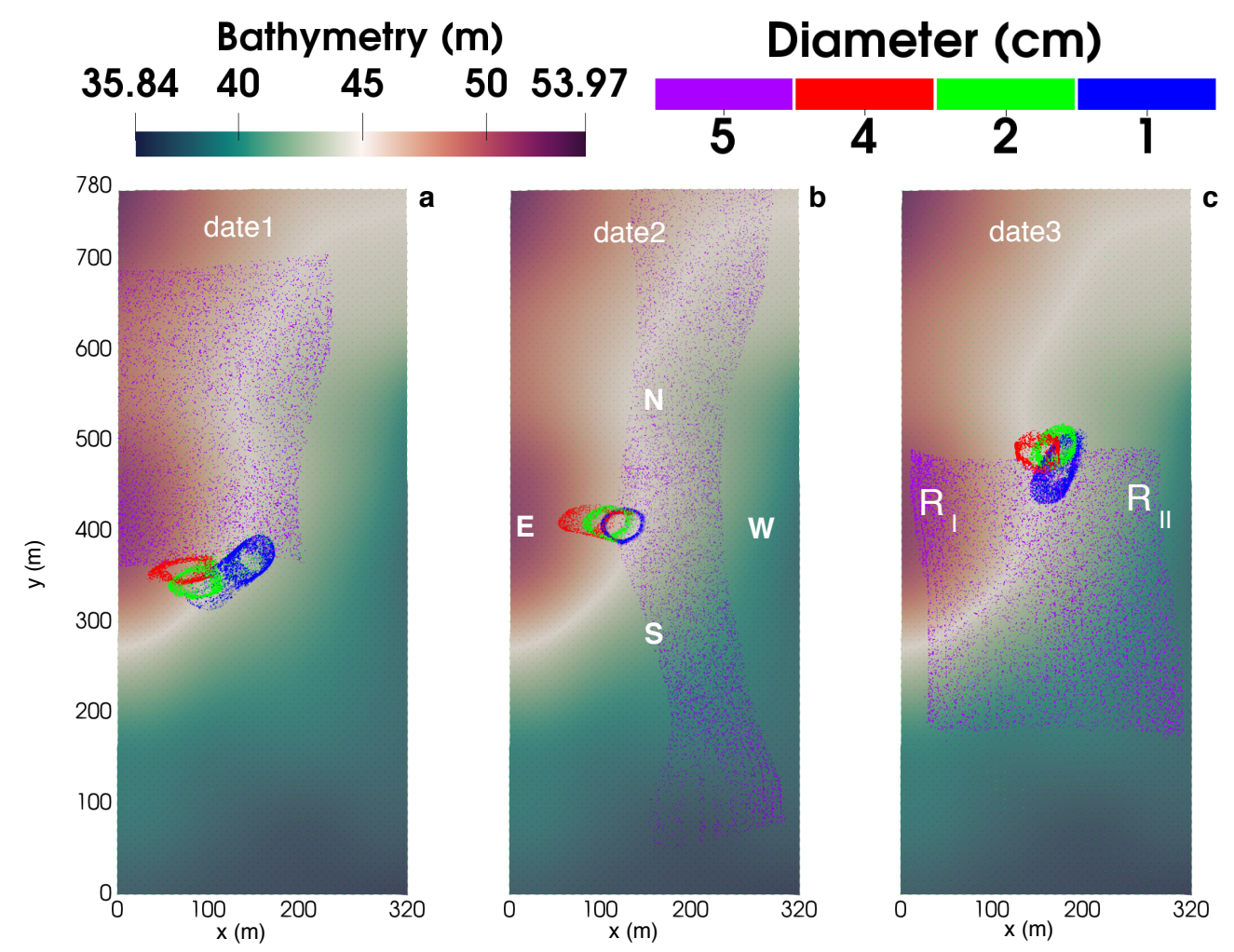

Figure 7: Effect of bed roughness coupling with ambient sediments using $t_{\text {init }}=$ date1 (a), date2 (b) and date 3 (c). The particle motion is dominated by the seabed morphology in the region $R_{I}$ and by the flow direction in the region $R_{I I}$.
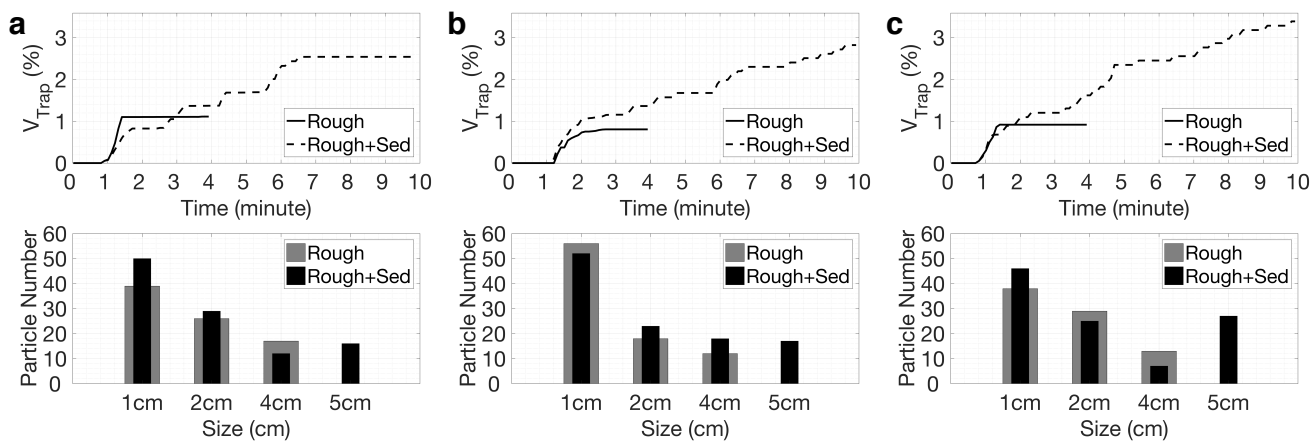

Figure 8: Time evolution of $\mathbf{V}_{\text {Trap }}$ with drilling residuals, bed roughness and ambient sediments (first row) and corresponding particle distribution inside the hole (second row). The results correspond to the three initial date presented in of Figure 7: date1 (first column), date2 (second column) and date3 (third column). 


\section{Ambient sediments}

We finally considered ambient sediments combined to drilling residuals and bed roughness effects. Influence of both seabed morphology and tidal currents direction are similar to the previous one:

- Case1: Combined effects of seabed morphology and Southwestward current (date 1)

At this date, the combined effect of the seabed morphology and tidal current leads to move sediments in the South-West direction as presented in Figure 7 (a). Therefore, the trapped sediments are those located near the bore hole and coming from the North-East direction. The interactions between residuals and sediments generate effects more important than for the smallest particles $(1 \mathrm{~cm}$ and 2 $\mathrm{cm}$ diameter) due to the high energy transfered during the shocks.

- Case2: Dominant effect of the seabed morphology (date 2)

During the reverse, the magnitude of tidal current is negligible and the sediments are essentially driven by the seabed morphology. Figure 7 (b) shows that, sediments and residuals are driven at East of the domain. The hole is then essentially filled by the sediments located near of the latter.

- Case3: Opposing effect between the seabed morphology and the Northeastward current (date 3)

The opposite effect between bathymetry variation and tidal direction leads to separate the displacement into two regions, denoted $R_{I}$ and $R_{I I}$ as presented in Figure 7 (c). The effect of bathymetry variation are dominant in the region $R_{I}$, leading to move the ambient sediments in the Northeastward direction where the slope is more important. The flow dominates the effect of the seabed morphology in the region $R_{I I}$, leading to a motion of particles is Northwestward as described by the tidal current direction.

The corresponding filling volumes and particles distribution of case1, 2, and 3 are presented in Figure 8 (a)-(b) and (c) respectively. Configuration minimising the filling is for the case1, where we have a combined effects of seabed morphology and tidal currents.

\section{CONCLUSION}

We presented a numerical model to investigate the bore hole filling volume in Alderney Race. Considering only drilling residual over a smooth bathymetry, the results presented herein show that: (i) the direction of current plays a negligible role while the distance between the bore hole and the residuals remains smaller than twenty meters; (ii) the increase of this distance leads to reduce the filling volume. When a rough the bed is considered, contacts with a fixed particles cause a small increase of the filling volume. In addition, when ambient sediments are also considered, we show that the effects of the latter and the bed roughnesses are coupled with the effects of both seabed morphology and tidal currents direction according to three main cases of coupling: (i) Combined effects of seabed morphology and tidal current; (ii) Dominant effect of the seabed morphology; (iii) Opposing effects of seabed morphology and tidal current. This paper is the first application of the model to a coastal engineering problem. A potential second application is to use it to investigate the impact of ambient sediments on tidal converters.

\section{ACKNOWLEDGEMENTS}

F.Adong acknowledges the post-doctoral grant of CNRS in the framework of the ADEME project Pile $\&$ Tide. Results acquired with Ifremer MARS software. The authors acknowledge the Pôle de Calcul et de Données Marines (PCDM) for providing CAPARMOR (storage, computational resources, visualization, support services).

\section{References}

F. Adong, A. Bennis, and B. Mouaze. Numerical investigation of bore hole filling volume in a coastal area. Applied Ocean Research, in press.

P. Lazure and F. Dumas. An externalâ"internal mode coupling for a 3d hydrodynamical model for applications at regional scale (mars). Advances in Water Resources, 31(2):233 - 250, 2008. ISSN 0309-1708.

R. Maurin, J. Chauchat, B. Chareyre, and P. Frey. A minimal coupled fluid-discrete element model for bedload transport. Phys. Fluids, 27(11), 2015. 
V. Šmilauer et al. Yade Documentation 2nd ed. The Yade Project, 2015. http://yade-dem.org/doc/. 